\title{
Acinetobacter radioresistens
}

National Cancer Institute

\section{Source}

National Cancer Institute. Acinetobacter radioresistens. NCI Thesaurus. Code C86096.

A species of aerobic, Gram negative, rod shaped bacteria assigned to the phylum

Proteobacteria. This bacteria is nonmotile, non spore forming, catalase positive, oxidase and urease negative, highly resistant to gamma ray irradiation and hydrolyzes Tween 80.

A. radioresistens is commonly found in soil and cotton. 